\title{
Caracterização do Atendimento Psicológico na Saúde
}

\author{
Wilze Laura Bruscato* (1) \& Renata Pereira Condes (1) \\ Faculdade de Ciências Médicas da Santa Casa de São Paulo, São Paulo, SP, Brasil
}

\begin{abstract}
RESUMO - Este trabalho caracteriza as atividades realizadas pelo Serviço de Psicologia em um complexo de atenção à saúde prestador de serviços ao Sistema Único de Saúde (SUS). A utilização das intervenções foi medida pelo número absoluto e relativo de pacientes atendidos. A partir disso, foi identificado: perfil sociodemográfico, especialidade médica de origem, agente solicitante, motivos da solicitação e tipo de intervenção realizada. Um maior percentual de mulheres foi atendido, havendo o predomínio de quadros de ansiedade e depressão. As intervenções mais utilizadas foram a avaliação psicológica e o atendimento do familiar/cuidador. Estudos rotineiros de caracterização da assistência são essenciais para o monitoramento do desempenho dos serviços, permitindo verificar sua adequação e a necessidade de melhorias.
\end{abstract}

PALAVRAS-CHAVE: Sistema Único de Saúde, intervenção psicológica, psicoterapia, serviços de saúde

\section{Characterization of Psychological Care in Health}

\begin{abstract}
This paper characterizes the activities of the Psychology Service in a health care complex that provides services to the Single Health System (SUS). The use of interventions was measured by absolute and relative number of patients. It was identified: socio-demographic profile, medical specialty of origin, requesting agent, request the reasons, and type of intervention. A higher percentage of women were attended, and anxiety and depression were predominant. The most used interventions were psychological evaluation and interventions with family members/caregivers. Routine studies of characterization of care are essential for monitoring the performance of services, making it possible to verify their adequacy and the need for improvement.
\end{abstract}

KEYWORDS: Single Health System, psychological intervention, psychotherapy, health care services

Sofrimento e doença, bem como o processo de envelhecimento e a morte, fazem parte da existência humana. Os significados e os sistemas de explicação em relação a esses fenômenos naturais não se reduzem a evidências orgânicas e objetivas. Um indivíduo doente traz consigo, além do adoecimento, seus sentimentos, sua família, seu papel social e todas as implicações que a doença física colocou em sua vida. Existe uma ordem de significações psicológicas, sociais e culturais que influenciam as formas pelas quais cada pessoa experimenta os seus estados de saúde e doença, a expressão dos sintomas, assim como os hábitos e estilos de vida que condicionam as próprias práticas de atendimento à saúde.

A Psicologia, como ciência, e a subjetividade humana, como seu objeto de estudo, estão envolvidas em tais práticas. A Psicologia da Saúde, como campo de especialização da Psicologia, oferece contribuições para o entendimento da saúde e da doença, além de encorajar a integração das informações biológicas e sociais sobre saúde e doença com o atual conhecimento psicológico, facilitando a colaboração entre psicólogos e outros profissionais da área. Ela se ocupa da assistência, do ensino e da pesquisa sobre as conexões da Psicologia com todas as demais áreas da saúde. Ela se constitui como um campo de atuação que propicia a integração de conhecimentos na interface entre distúrbios orgânicos e manifestações psicológicas, através da priorização de um atendimento articulado multiprofissional. Ainda, ela aplica seus princípios, técnicas e conhecimentos científicos para avaliar, diagnosticar, tratar, modificar e prevenir os problemas físicos, mentais ou qualquer outro relevante para os processos de saúde e doença. Essa atuação profissional pode ser realizada em ambientes distintos e variados, como hospitais, ambulatórios, hospitais-dia, prontos-socorros, centros de saúde, domicílios e na própria comunidade (Bruscato, 2012; Castro \& Bornholdt, 2004; Matarazzo, 1982).

\footnotetext{
*E-mail: wlbruscato@uol.com.br

- Submetido: 16/09/2015; Revisado: 18/04/2016; Aceito: 09/05/2016.
} 
Nesses espaços, a Psicologia da Saúde resgata o ser humano para além de sua dimensão físico-biológica para situá-lo num contexto maior de sentido e significado na sua dimensão psicológica. Recupera a singularidade do paciente, suas emoções, crenças e valores, numa relação dialética com a cultura à qual pertence, definindo seu campo de atuação, sua contribuição teórica efetiva e possibilitando o desenvolvimento de modelos de atuação adaptados às necessidades de saúde das populações atendidas (Bruscato, 2006, 2012; Traverso-Yépez, 2001;).

Os notáveis avanços na área da saúde nas últimas décadas, que trouxeram mudanças no processo do adoecer, com a diminuição de doenças infecciosas decorrente de medidas preventivas e o aumento das doenças crônicas, também evidenciaram o reconhecimento da própria responsabilidade do indivíduo nos cuidados e a importância dada aos fatores comportamentais como determinantes na mudança de hábitos e costumes, que podem levar à doença ou à manutenção da saúde. Também possibilitaram uma inegável melhoria da qualidade de vida do doente, contribuíram para modificar a relação do homem com seu corpo e com sua vida e tornaram mais claro o papel dos aspectos psicológicos no âmbito das práticas de saúde. Entretanto, a instituição de saúde ainda é marcada por situações extremas, por sofrimento, por dor e pela luta constante entre vida e morte. No adoecimento, potencializam-se angústias, medos, inseguranças, raivas e revoltas, não só para doentes e familiares como também para o próprio profissional da saúde, sempre preparado para a cura, mas em constante tensão diante da morte (Amaral et al., 2012).

A existência de disfunções psicológicas e comportamentais deflagradas por diferentes quadros clínicos ou cirúrgicos é notória e amplamente documentada na literatura. Vários estudos nacionais estimaram a prevalência de distúrbios psicológicos nos serviços de saúde, que oscila entre $28,7 \%$ e $50 \%$ e é considerada alta, em especial para o sexo feminino e para os idosos (Borim et al., 2013; Gazotti \& Prebianchi, 2014; Moreira et al., 2011; Ribeiro et al., 2006). Em um estudo de Lucchese et al. (2014), com pacientes da Atenção Primária da Região Centro-Oeste, 31,5\% apresentaram problemas emocionais ligados à questão da saúde, com maior prevalência no sexo feminino, idade entre 18 e 59 anos, ocupação do lar, com filhos, com quatro a sete anos de estudo e renda de até um salário mínimo. Catani et al. (2012), ao pesquisarem sobre os encaminhamentos para o serviço de Psicologia, encontraram que $71 \%$ dos pacientes eram mulheres e a faixa etária dos 30 aos 50 anos apresentou uma maior concentração, representando $65 \%$ do total.

Os dados gerados por esses estudos aproximam-se dos encontrados na literatura internacional, que refere que entre um terço e metade das pessoas atendidas ambulatorialmente por um clínico apresentam dificuldades emocionais e cerca de $60 \%$ dos pacientes internados apresentam algum tipo de distúrbio psicológico, que pode ser um problema crônico, uma manifestação decorrente do quadro clínico de base ou, ainda, reações à doença aguda, ao seu tratamento e à hospitalização. Tradicionalmente, segundo a literatura, a população atendida por psicólogos nesses serviços de saúde caracteriza-se majoritariamente como feminina, entre 20 e 45 anos (64\%) e com predominância de quadros ansiosos e depressivos (58\%) (Botega \& Smaira, 2012; Bruscato, 2006).

Assim, os pacientes com depressão, ansiedade e distúrbios de ajustamento constituem a parcela numericamente mais significativa dos casos atendidos em serviços de Psicologia nos equipamentos de saúde. Alguns desses problemas são transitórios, secundários às condições físicas dos pacientes e à internação hospitalar, tendendo a remitir com a melhora do quadro clínico e a alta hospitalar. Outros podem ser mais graves, a ponto de causar considerável sofrimento e de prejudicar a evolução do paciente (Botega, 2012).

Além dos quadros de ansiedade e depressão, alguns autores referem a ocorrência de problemas psicossomáticos nos quais a expressão de sofrimento emocional se daria através de sensações corporais. O psicólogo exerceria então a função de "decodificador", fazendo a ponte entre o somático e o psíquico (Botega \& Smaira, 2012; Bruscato, 2006).

A maioria dos estudos se refere aos diagnósticos mais frequentemente atendidos por esses Serviços de Psicologia, independentemente de onde se originou o pedido. Contudo, quanto ao local de origem do pedido, a demanda por atendimento psicológico pode ser vista na literatura como se originando, em sua grande maioria, da clínica médica, destacando-se também a cardiologia, a clínica da dor, a dermatologia, a reumatologia, a ginecologia, a pediatria, quadros de AIDS e a oncologia (Fortes, 2012; Kitayama \& Bruscato, 2008). Pacientes das clínicas neurológicas também demandam atendimento psicológico pela necessidade do uso de métodos especializados de investigação neuropsicológica (Braga et al., 2012). Alguns estudos referem a assistência psicológica nas questões ligadas aos transplantes (Garcia \& Zimmerman, 2012) e ao paciente na Unidade de Terapia Intensiva (UTI; Zimmerman \& Bertuol, 2012). No caso dos transplantes, conforme a evolução técnica foi ocorrendo, surgiu a necessidade de se observarem os aspectos psicológicos e psicossociais dos pacientes transplantados e seus familiares. O evidente aumento das taxas de sobrevida desses pacientes abriu as portas para pesquisas acerca da qualidade de vida dessas pessoas.

Quanto à UTI, considera-se que ela é, em si, um fator precipitante de problemas psicológicos. O paciente internado na UTI, além de apresentar um quadro clínico grave, está submetido à dor, ao sofrimento, à solidão e ao medo da morte. Além disso, a UTI é um ambiente gerador de stress contínuo para o médico e sua equipe. Para os familiares, ela é, sem dúvida, uma das maiores fontes de ansiedade, principalmente pela sensação de impotência, pela vulnerabilidade a que o paciente fica submetido e pelo grande número de informantes (médicos, residentes, plantonistas, enfermeiros, auxiliares de enfermagem) (Haberkorn \& Bruscato, 2008). 
Todos esses dados justificam a relevância de ações de caracterização de casos de distúrbios psicológicos nos serviços de saúde, desde o âmbito da Atenção Primária até a alta complexidade. A equipe de Psicologia monitora frequentemente os atendimentos para atualizar o conhecimento da solicitação pelo serviço e planejar, redirecionar ou adequar a prestação de serviço psicológico.
Dessa forma, é possível identificar a demanda, descrever o perfil dos usuários, conhecer os motivos e necessidades da população e avaliar a correspondência entre a procura e a oferta do atendimento na tentativa de aproximar as necessidades à prestação de serviço (Baremblitt, 2002; Vanni \& Maggi, 2005).

\section{OBJETIVO}

Caracterizar o atendimento psicológico nas diferentes unidades de um complexo de cuidados de atenção à saúde em todos os níveis de atenção estabelecidos pelo
SUS, descrevendo o perfil dos usuários, identificando os motivos e origens da demanda e avaliando a oferta do atendimento.

\section{MÉTODO}

Trata-se de um estudo transversal descritivo, realizado entre junho e dezembro de 2013, inserido na Plataforma Brasil, com parecer favorável da Comissão de Ética em Pesquisa $n^{0} 921.621$ e aprovado sob $n^{\circ}$ CAAE 37940114.9.0000.5479. Um protocolo para registro dos dados para caracterização do perfil dos pacientes nas diversas áreas de atuação da Psicologia foi elaborado com base no material de uso de rotina do Serviço de Psicologia. O protocolo era composto por 13 variáveis: Idade do paciente, Sexo, Raça/cor, Escolaridade, Estado civil, Situação ocupacional, Clínica de origem, Atendimento por outra especialidade, Categoria profissional solicitante, Motivo da solicitação, Queixa do paciente, Diagnóstico psicológico e Intervenção realizada e Conduta. Os dados eram registrados a cada atendimento e foram inseridos no banco de dados do Excel, utilizado para a análise descritiva das frequências.

\section{Caracterização do Campo Pesquisado}

Quando da instauração do Sistema Único de Saúde (SUS), o desenvolvimento da diretriz da hierarquização segmentou o sistema de saúde em níveis de complexidade como uma forma de organizar os serviços e as ações para atender às diferentes necessidades de saúde da população (Brasil, 1990). Quando nos referimos a níveis de atenção, estamos tratando da capacidade que uma unidade de saúde tem para prestar assistência. Essa capacidade se organiza em (a) Primária ou Básica, de atenção generalista, com prestação de serviços voltados para o atendimento das necessidades mais comuns e frequentes, de baixa complexidade tecnológica, com foco na promoção e na prevenção; (b) Secundária, com ações e serviços que visam a atender problemas de saúde e agravos da população, cuja prática clínica demande profissionais especializados e uso de recursos tecnológicos de apoio diagnóstico e terapêutico em um nível médio de complexidade; (c) Terciária, com utilização de tecnologia de alto nível e alto custo, além da realização de procedimentos de alta complexidade e atendimento muito especializado, necessários para situações mais graves, que exigem serviços com equipamentos e profissionais com maior potencial de resolução. Atualmente fala-se também em Atenção Quaternária, relacionada ao risco de adoecimento iatrogênico, ou seja, provocado por atenção inadequada à saúde, como o excessivo intervencionismo diagnóstico terapêutico, além da medicalização desnecessária e sugestão de intervenções eticamente aceitáveis.

A instituição na qual foi realizado este estudo é uma entidade privada e filantrópica prestadora de serviços ao SUS, em todos os níveis de atenção. No ano de 2013, sua disponibilidade assistencial era representada por 2.504 leitos e registrou 48.962 cirurgias, internando 763.774 pacientes, o que equivale a 2.092 por dia. Houve 1.601 .812 atendimentos ambulatoriais, 2.013.704 atendimentos de emergência e foram realizados 6.238.439 exames (SISC, 2015). Nesse ano, esse serviço atendeu mais de $10 \mathrm{mil}$ pessoas por dia, incluindo atendimentos em enfermarias, ambulatórios, domicílios, hospitais-dia, centros de saúde, serviços de emergência e serviços de terapia intensiva, prestando, através da assistência multiprofissional integrada, cuidados de atenção à saúde em todos os níveis de atenção estabelecidos pelo SUS, em diferentes especialidades médicas.

Os profissionais do Serviço de Psicologia da instituição, que eram $80 \mathrm{em}$ quantidade total na ocasião, prestam atendimentos psicodiagnósticos e também serviços de orientação, de reabilitação e psicoterapêuticos para crianças, adolescentes, adultos e idosos, pacientes ou familiares e cuidadores, profissionais ou trabalhadores da saúde nas diferentes Unidades/Clínicas/Serviços da instituição, dentro de equipes de todas as áreas da saúde e em diversas situações de rotina, desde a atenção primária até a alta complexidade.

Em todas as unidades estudadas, o trabalho está calcado no pressuposto de que o usuário que precisa de cuidados 
em saúde mental necessita naquele momento e, com isso, as portas de entrada estão abertas para que esses usuários acessem o profissional da Psicologia. Isto é a organização da entrada no serviço é peça fundamental no cumprimento aos princípios de igualdade, universalidade e integralidade pressupostos pelo SUS e no acolhimento aos usuários que necessitem de suporte de um profissional de saúde mental. Manter um serviço disponível aos usuários não é uma tarefa fácil, mas o desafio é parte do trabalho de busca de tecnologias em saúde, organizando-o e reorganizando-o sempre que necessário. É uma construção permanente para os profissionais.

O psicólogo, ao atender usuários dos serviços de saúde, é parte de uma equipe multiprofissional, pratica intervenções em consonância com os demais colegas e considera o trabalho conjunto, as trocas interdisciplinares, as discussões diagnósticas, os encaminhamentos pertinentes, as possibilidades e os limites de cada intervenção, visando ao melhor resultado no atendimento ao paciente. Parte-se do princípio de que o enfoque global do paciente pressupõe não apenas a somatória das diversas práticas, mas sua integração através da construção coletiva de um saber mais amplo e próprio de uma equipe. O trabalho multiprofissional é uma possibilidade de ampliar a capacidade humana de compreender a realidade e os problemas que nela se apresentam, levando em conta a interdependência dos saberes para a execução e o cumprimento do mesmo objetivo. Esse trabalho se refere à composição de diferentes processos, à flexibilização e à divisão do trabalho, preservando as diferenças técnicas entre os trabalhadores especializados, mas diminuindo as desigualdades na valorização dos distintos trabalhos e respectivos agentes, bem como nos processos decisórios (Oliveira et al., 2011).

Toda a prática profissional dos psicólogos é vinculada ao SUS. Na atenção primária, forma tecnologicamente específica de atenção generalista, a prestação de serviços psicológicos é voltada para o atendimento de casos instrumentalmente simples, que não necessitam de aparatos ou alta tecnologia para o diagnóstico. A característica primordial da atenção primária é ser a porta de entrada para o sistema de saúde, tendo, como atributos essenciais e interrelacionados na prática clínica, o acesso de primeiro contato do indivíduo com o sistema, a continuidade do cuidado, a integralidade da atenção e a coordenação do cuidado dentro do sistema, o que aumenta seu poder de interação com os indivíduos e com a comunidade. Apresenta, ainda, outras características que qualificam as ações: a atenção à saúde centrada na família, a orientação comunitária e a competência cultural.

Os atendimentos secundários, terciários e quaternários são realizados em ambulatórios, enfermarias, prontossocorros, pronto atendimento e UTIs, tanto para o paciente como para os familiares, através de uma diversidade de modalidades de atuação: triagem, psicodiagnóstico, atendimento psicoterapêutico individual ou grupal, grupos de profilaxia, grupos de reflexão, intervenção pré e póscirúrgica e atendimento familiar, seguindo programas específicos em parceria com as especialidades médicas. Esses atendimentos têm um caráter informativo, profilático e terapêutico, sendo que alguns deles contam com a participação de outros profissionais da área da saúde. $\mathrm{O}$ atendimento pode acontecer por meio do Sistema de Ligação, no qual o psicólogo atua como membro da equipe, ou por Sistema de Consultoria, em que atende à solicitação do paciente, do familiar, docuidador ou da equipe, mediante pedido de consulta. No Sistema de Ligação, a presença do psicólogo é permanente e ele passa a ser um membro efetivo da equipe daquele local, atendendo seus pacientes, participando das reuniões clínicas e lidando com aspectos da relação estabelecida entre equipe, paciente e família. No Sistema de Consultoria, o profissional da Psicologia avalia, indica e/ou realiza um tratamento para o paciente que está sob os cuidados de outros especialistas. A presença desse profissional é episódica, respondendo a uma solicitação específica vinda de uma clínica ou serviço. Também ocorrem solicitações vindas diretamente do paciente ou de seus familiares que, espontaneamente, procuram pelo atendimento psicológico (Amaral et al., 2012). Seja qual for a modalidade de atendimento, o paciente chega ao psicólogo por demanda espontânea, por solicitação de familiar ou pelo encaminhamento de qualquer outro profissional da equipe.

Todos os psicólogos mantêm um registro das ações através do uso de protocolos de avaliação, evolução e acompanhamento padronizados, tendo totalizado 52.399 atendimentos no ano de 2013. O psicólogo nessa instituição tem, então, uma função clínica, social, institucional e educacional, através de uma assistência psicológica regulada pela urgência e flexibilidade, que inclui, como clientela, não só o paciente, mas também seus familiares, cuidadores, profissionais e trabalhadores da saúde e equipes. Ele seleciona e prioriza atendimentos que estejam de acordo com as possibilidades e demandas, como decorrência de estudos científicos do que ocorre nas populações assistidas e que apontam para técnicas de intervenção mais eficazes (Bruscato, 2012).

\section{RESULTADOS}

Levando em conta a pluralidade e diversidade dos equipamentos de saúde da instituição, o intuito deste estudo foi caracterizar o serviço prestado como um todo, porém sem desconsiderar que cada unidade tem uma população diferenciada, com necessidades específicas. Entre junho e dezembro de 2013, foram atendidos pelos psicólogos 5.893 
pacientes, que geraram 32.478 atendimentos $(62 \%$ dos 52.399 atendimentos realizados no ano de 2013). Destes, 923 $(15,7 \%)$ foram atendidos nas unidades de Atenção Primária e os demais (4.970, que representam 84,3\%) nos equipamentos de saúde do complexo, que atendem os níveis de atenção secundária, terciária e quaternária: hospitais gerais, com todas as especialidades médicas, 4.226 pacientes $(71,7 \%)$; unidades de saúde mental, 526 pacientes $(8,9 \%)$; unidades de neurologia, 218 pacientes $(3,7 \%)$.

Do total, $2.560(43,4 \%)$ eram do sexo masculino, 3.332 $(56,5 \%)$ do sexo feminino e 1 paciente $(0,02 \%)$ se declarou transexual. Quanto à idade, $1.285(21,8 \%)$ tinham entre 0 e 18 anos; 3.217 (54,6\%) tinham entre 19 e 60 anos; 1.295 (22\%) tinham de 61 a 90 anos; 71 pacientes $(1,2 \%)$ tinham mais de 90 anos. Para 25 pacientes $(0,4 \%)$, não foi possível aferir a idade. A maior concentração de pacientes (852) estava entre os 30 e os 40 anos (14,5\%). Quanto à raça/ cor autorreferida, $3.352(56,9 \%)$ declararam-se brancos; $2.170(36,8 \%)$, afrodescendentes; $106(1,8 \%)$, amarelos; e $265(4,5 \%)$ não referiram raça ou cor. A escolaridade dos pacientes estava assim distribuída: $548(9,3 \%)$ eram analfabetos; 2.290 (30,9\%) estavam no Ensino Fundamental; 1.518 (25,8\%), no Ensino Médio/Técnico; 684 (11,6\%), no Ensino Superior; e 34 pacientes $(0,6 \%)$ tinham alguma pós-graduação. Para 819 pacientes $(13,9 \%)$, que incluem os $522(8,9 \%)$ com idade de zero até 6 anos, não foi possível obter esse dado. Quanto ao estado civil, 2.423 (41,1\%) eram solteiros, 2.466 (41,8\%), casados ou em união estável; 509 $(8,6 \%)$, separados ou divorciados e 408 (6,9\%), viúvos. Por outro lado, 87 (1,5\%) pacientes não forneceram esse dado. A situação ocupacional se distribuía assim: 857 (14,5\%) estudantes; 1.263 (21,4\%) com vínculo empregatício; 650 (11\%) desempregados; 485 (8,2\%) com atividade autônoma; $708(12 \%)$ realizavam atividades domésticas; $486(8,3 \%)$ estavam em licença médica; 774 (13,1\%) eram aposentados; e $670(11 \%)$ eram ou crianças abaixo da faixa de escolaridade ou não referiram esse dado.

Quando investigamos a clínica de origem da solicitação de consulta para a Psicologia, $2.095(35,6 \%)$ eram oriundos das UTIs; 708 (12\%), da Psiquiatria; 685 (11,6\%), da Clínica Médica; 437 (7,4\%), da Clínica Cirúrgica; 289 (4,9\%), da Ortopedia, Traumatologia e Serviço de Reabilitação Física; 269 (4,6\%), da Pediatria e Puericultura; 234 (4\%), da Ginecologia e Obstetrícia; 205 (3,5\%), do Serviço de Segurança e Medicina do Trabalho (SESMT); 151 (2,6\%), da Neurologia e Neurocirurgia; 9 (0,2\%), da Geriatria. Tiveram demanda espontânea 230 pacientes $(3,9 \%)$. Tiveram outras origens $581(9,9 \%): 198(3,4 \%)$ vieram de encaminhamento externo e $383(6,5 \%)$ de outras especialidades não especificadas. Mais da metade dos pacientes $(3.112$ ou $52,8 \%$ ) eram atendidos em mais de uma especialidade médica. A categoria profissional solicitante ficou assim distribuída: $3.420(58 \%)$ encaminhamentos foram feitos pelo médico; 544 (9,2\%), pelo psicólogo; $225(3,8 \%)$, por enfermeiros ou auxiliares de enfermagem; 133 (1,9\%), por assistentes sociais, 196 (3,3\%), pela Equipe de Saúde da Família; e $1.395(23,7 \%)$, por outras categorias profissionais (fisioterapeuta, fonoaudiólogo, terapeuta ocupacional, nutricionista e outras). Dentre os motivos da solicitação de atendimento psicológico, os mais frequentes foram: 1.269 $(21,5 \%)$ quadros de depressão (451 ou 7,7\%); ansiedade (418 ou 7,1\%); ambos (400 ou 6,8\%); $429(7,3 \%$ ) pedidos por comportamento desadaptado, sem adesão ao tratamento e com geração de conflito de relacionamento com a equipe; e 268 (4,5\%) solicitações para preparo e acompanhamento pré e pós-cirúrgico.

Quando avaliamos a queixa do paciente, os resultados mostraram que mais da metade dos pacientes (3.188 ou $54,1 \%)$ referiu ansiedade e/ou depressão: 1.584 (26,9\%) referiam ansiedade; 1.022 (17,3\%), ansiedade e depressão; e 582 (9,9\%), depressão. O diagnóstico psicológico trouxe como principais resultados: $57,1 \%$ dos pacientes foram diagnosticados com ansiedade ou depressão: 2.359 (40\%) pacientes com ansiedade e $1.066(17,1 \%)$ com depressão. Além destes, os pacientes também apresentavam, algumas vezes em comorbidade: transtorno do ajustamento (1.527 ou 25,9\%); não compreensão ou não aceitação da doença, com consequente não adesão ao tratamento e desadaptação à situação ( 1.520 ou $25,8 \%$ ); conflitos de relacionamento com equipe ou familiares (1.165 ou 19,8\%). Também foram encontrados, em menor proporção, casos de outros transtornos mentais ou cognitivos e dificuldades emocionais diversas, casos de abuso, negligência, maus-tratos e violência doméstica.

As intervenções realizadas e condutas foram: 4.466 pacientes foram submetidos a avaliações psicológicas ou neuropsicológicas $(75,8 \%) ; 3.313(56,2 \%)$ pacientes receberam atendimento psicoterápico; 4.587 (77,8\%) dos familiares ou cuidadores receberam orientação ou atendimento; $\mathrm{e}$ as equipes foram orientadas quanto à melhor abordagem para atender $2.138(36,3 \%)$ pacientes. Esses dados podem ser vistos na Tabela 1 .

O perfil padrão da população estudada foi o de um indivíduo do sexo feminino, idade entre 19 e 60 anos, branco, escolaridade de Ensino Fundamental, casado ou em união estável e situação ocupacional de vínculo empregatício. Era oriundo de uma Unidade de Terapia Intensiva, sendo atendido por mais de uma especialidade médica. A solicitação de atendimento foi feita pelo médico, tendo, como motivo, ansiedade ou depressão, o que condizia com a sua própria queixa, sendo confirmado por diagnóstico psicológico. 
Tabela 1

Variáveis de caracterização do perfil sociodemográfico dos pacientes atendidos e dos atendimentos

\begin{tabular}{|c|c|}
\hline Variáveis & $\mathbf{N}(\%)$ \\
\hline 1) Gênero & $\begin{array}{l}\text { Masculino }=2.560(43,4 \%) \\
\text { Feminino }=3.332(56,5 \%)\end{array}$ \\
\hline 2) Idade & $\begin{array}{l}0 \text { a } 18 \text { anos }=1.285(21,8 \%) \\
19 \text { a } 60 \text { anos }=3.217(54,6 \%) \\
61 \text { a } 90 \text { anos }=1.295(22 \%) \\
>90 \text { anos }=71(1,2 \%)\end{array}$ \\
\hline 3) Raça/cor & $\begin{array}{l}\text { Branca }=3.352(56,9 \%) \\
\text { Afrodescendente }=2.170(36,8 \%) \\
\text { Amarela }=106(1,8 \%) \\
\text { Não referida }=265(4,5 \%)\end{array}$ \\
\hline 4) Escolaridade & $\begin{array}{l}\text { Analfabeto }=548(9,3 \%) \\
\text { Ensino Fundamental }=2.290(30,9 \%) \\
\text { Ensino Médio/Técnico }=1.518(25,8 \%) \\
\text { Ensino Superior }=684(11,6 \%) \\
\text { Pós-Graduação/Especialização }=34(0,96 \%) \\
\text { Sem dados }=819(13,9 \%)\end{array}$ \\
\hline 5) Estado civil & $\begin{array}{l}\text { Solteiro }=2.423(41,1 \%) \\
\text { Casado/União estável }=2.466(41,8 \%) \\
\text { Separado/Divorciado }=508(8,6 \%) \\
\text { Viúvo }=408(6,9 \%) \\
\text { Sem dados }=87(1,5 \%)\end{array}$ \\
\hline 6) Situação Ocupacional & $\begin{array}{l}\text { Estudante }=857(14,5 \%) \\
\text { Vínculo empregatício }=1.263(21,4 \%) \\
\text { Desempregado }=650(11 \%) \\
\text { Autônomo }=485(8,2 \%) \\
\text { Atividade doméstica }=708(12 \%) \\
\text { Licença médica }=486(8,35) \\
\text { Aposentado }=774(13,1 \%) \\
\text { Sem dados }=670(11 \%)\end{array}$ \\
\hline 7) Clínica de origem & $\begin{array}{l}\text { Unidade de Terapia Intensiva }=2.095(35,6 \%) \\
\text { Psiquiatria }=708(12 \%) \\
\text { Clínica Médica }=685(11,6 \%) \\
\text { Clínica Cirúrgica }=437(7,4 \%) \\
\text { Ortopedia, Traumatologia, Reabilitação Física = } 289(4,9 \%) \\
\text { Pediatria e Puericultura }=269(4,6 \%) \\
\text { Ginecologia e Obstetrícia }=234(4 \%) \\
\text { Serviço de Segurança e Medicina do Trabalho = } 205(3,5 \%) \\
\text { Neurologia e Neurocirurgia }=151(2,6 \%) \\
\text { Geriatria }=9(0,2 \%) \\
\text { Demanda espontânea }=230(3,9 \%) \\
\text { Outros }=581(9,9 \%)\end{array}$ \\
\hline 8) Atendimento em outra especialidade & $\operatorname{Sim}=3.112(52,8 \%)$ \\
\hline 9) Categoria Profissional solicitante & $\begin{array}{l}\text { Médico }=3.420(58 \%) \\
\text { Psicólogo }=544(9,2 \%) \\
\text { Enfermeiro/Auxiliar de Enfermagem = } 225(3,8 \%) \\
\text { Assistente Social }=133(1,9 \%) \\
\text { Equipe de Saúde da Família = } 196(3,3 \%) \\
\text { Outras }=1.395(23,7 \%)\end{array}$ \\
\hline 10) Motivo da solicitação & $\begin{array}{l}\text { Depressão }=451(7,3 \%) \\
\text { Ansiedade }=418(7,1 \%) \\
\text { Ansiedade e depressão }=400(6,8 \%) \\
\text { Comportamento desadaptado }=429(7,3 \%) \\
\text { Preparo e acompanhamento pré e pós-cirúrgico }=268(4,5 \%)\end{array}$ \\
\hline 11) Queixa do paciente & $\begin{array}{l}\text { Ansiedade }=1.584(26,9 \%) \\
\text { Depressão }=582(9,9 \%) \\
\text { Ansiedade e depressão }=1.022(17,3 \%)\end{array}$ \\
\hline
\end{tabular}




\begin{tabular}{ll}
\hline Variáveis & \multicolumn{1}{c}{$\mathbf{N}(\mathbf{\%})$} \\
\hline & Ansiedade $=2.359(40 \%)$ \\
& Depressão $=1.066(17,1 \%)$ \\
& Transtorno do ajustamento $=1.527(25,9 \%)$ \\
& Não compreensão/nãoaceitação da doença $=1.520(25,8 \%)$ \\
& Conflitos com a equipe ou familiares $=1.165(19,8 \%)$ \\
& Avaliação psicológica/neuropsicológica $=4.466(75,8 \%)$ \\
& Atendimento psicoterápico $=3.313(56,2 \%)$ \\
13) Intervenção realizada e conduta & Atendimento ou orientação ao familiar/cuidador $=4.587(77,8 \%)$ \\
& Orientação à equipe $=2.138(36,3 \%)$ \\
\hline
\end{tabular}

\section{DISCUSSÃO}

Buscamos estudos feitos com a população que utiliza o SUS, pela possibilidade mais próxima de comparação com os dados que obtivemos. O SUS é o maior agente de financiamento de atenção à saúde do país e, ao estabelecer como diretriz o acesso universal e integral à atenção à saúde, propõe um desafio: atender a uma população de mais de 200 milhões de pessoas, tanto no provimento de ações básicas quanto no de maior complexidade (IBGE, 2015; RIPSA, 2015; Silva et al., 2011). O estudo, a caracterização e a compreensão da utilização dos serviços na saúde é uma boa ferramenta para medir o acesso à assistência nessa área $e$ isso inclui também os serviços de assistência psicológica.

Estudos afirmam que a efetiva utilização decorre de uma complexa combinação de fatores, que incluem desde a necessidade, a percepção, as características sociodemográficas e os valores do indivíduo até a organização e a forma de funcionamento da instituição de saúde (Mendoza-Sassi et al., 2003; Travassos \& Martins, 2004). Em pesquisas realizadas sobre a utilização de serviços de saúde de um modo geral, as variáveis foram: tipo de atendimento, tipo de serviço e motivos referidos da procura pelo serviço. O perfil dos usuários foi identificado a partir de variáveis como sexo, idade, cor da pele, escolaridade, renda familiar e região de residência (Ruiz, 2015; Silva et al., 2011). Os principais resultados revelam que 52,4\% dos atendimentos foram feitos por outros profissionais da área da saúde que não o médico ou o enfermeiro, dentre os quais está o psicólogo. O tipo de serviço mais utilizado foi o atendimento hospitalar secundário e terciário $(75,2 \%)$, o que corrobora nossos achados de $84,3 \%$ da utilização dos serviços psicológicos na média e alta complexidade. Entendemos que há, nesses níveis de atenção, uma maior oferta de serviços, o que explica essa maior utilização. Houve mais uso desses serviços por mulheres (57\%) que se posicionavam no extremo inferior de escolaridade.

Diversos outros estudos específicos de atendimento psicológico na área da saúde confirmam a maior proporção de mulheres (de 61,7\% a 71\%), a escolaridade de Ensino Fundamental $(41,9 \%)$ e um aumento de procura em função da idade (de $59,8 \%$ a $65 \%$ entre 15 e 59 anos) e das pessoas de cor branca $(57,2 \%$ contra $42,8 \%$ de pretos e pardos), como nos nossos achados (Catani et al., 2012; Gazotti \& Prebianchi, 2014; Ribeiro et al., 2006;).

Em outras pesquisas que analisavam o perfil sociodemográfico e a satisfação dos usuários de serviços de Fisioterapia em Teresina (PI) (Machado \& Nogueira, 2008), assim como neste estudo, a maioria dos usuários era de mulheres $(62,5 \%)$, com idade na faixa dos 50 anos e casadas (63\%). Michelone e Santos (2004), ao analisarem as características sociodemográficas de pacientes com câncer, também revelam maior proporção de casados ou com companheiro $(64,5 \%)$.

Não foram encontrados dados confiáveis quanto à situação ocupacional. Talvez por ser uma variável que se altera, uma vez que os pacientes podem apresentar uma situação ao serem admitidos que se modifica no decorrer do tratamento.

No que diz respeito à clínica de origem, Gazotti e Prebianchi, (2014) referem a Ginecologia $(21,6 \%)$ e a Clínica Médica (12,6\%). Em outro estudo, Santoset al. (2011) trazem também a Clínica Médica (19\%). Não foram encontrados estudos de caracterização que incluíssem UTIs como o local de maior quantidade de encaminhamentos como neste estudo. Podemos entender essa maior demanda na nossa instituição, levando em conta que o desenvolvimento tecnológico no diagnóstico e tratamento de inúmeras doenças, o aumento da expectativa de vida da população geral e a sobrevida de pacientes com doenças outrora letais levaram a um acréscimo da demanda de pacientes para UTI, que é um recurso disponível na média e na alta complexidade para fornecimento dos cuidados necessários, apresentando resultados positivos para manutenção da saúde e prolongamento da vida (Chiavone \& Sens, 2003; Haberkorn, 2006). Entretanto, a internação na UTI, que coloca a possibilidade de perder a vida ou de prolongá-la indefinidamente, afastando a morte, é considerada um dos momentos mais críticos e amedrontadores no processo de hospitalização. Essa unidade é fonte contínua de angústias, 
incertezas e medos, bem como um lugar para o qual se acredita ir para morrer (Haberkorn \& Bruscato, 2008).

Os ruídos, os estímulos dolorosos, a iluminação artificial constante, a estimulação sonora dos aparelhos, a restrição do campo visual, a movimentação contínua da equipe, a privação do sono, a agitação do ambiente, a mecanização do cuidado, a falta de privacidade em atividades rotineiras de higiene, o afastamento do doente de seus familiares, a perda do contato com o ambiente social e profissional, o contato com pessoas desconhecidas, a perda da independência e da autonomia sobre o próprio corpo e o não entendimento da situação levam o doente a apresentar sentimentos de insegurança, desamparo, isolamento, solidão e desesperança. Sobretudo, também é uma situação de medo, tanto da dor quanto de grandes sofrimentos físicos, de mutilação ou de dano a partes do corpo, da perda do controle sobre a própria vida e, principalmente, da morte (Haberkorn, 2006; Zimmermann \& Bertuol, 2012).

Especificamente com relação ao atendimento psicológico do paciente internado em UTI, há demanda para aqueles que se apresentam ansiosos, deprimidos, com agitação psicomotora, agressivos, amedrontados, chorosos, pouco colaborativos com os procedimentos, desorientados, com ideação suicida e em situação de terminalidade. É atribuída ao psicólogo a tarefa de particularizar e individualizar o atendimento, dar sentido e amenizar, de forma terapêutica, os sentimentos, as fantasias e as dificuldades provindas dessa situação estressante e, com isso, permitir o alívio das angústias e a melhora da qualidade da permanência, levando em conta a "urgência psicológica" em que se encontram o paciente e sua família pelo sofrimento (Haberkorn \& Bruscato, 2008).

Quanto ao agente solicitante, os estudos encontrados na literatura indicam que, na maioria das vezes, $o$ atendimento psicológico acontece por indicação ou solicitação da equipe médica ou de enfermagem (Osório, 2004). Santos et al. (2011) referem que $44 \%$ das solicitações foram feitas por médicos e $38 \%$ por enfermeiros. Essa relação o fica invertida nos estudos de Gazotti e Prebianchi (2014), cujos achados referem $51,4 \%$ de solicitações por parte dos enfermeiros e $18 \%$ dos médicos. Em nossa caracterização, o principal solicitante foi o médico (58\%). O presente trabalho não teve o objetivo de identificar que elementos induzem ao encaminhamento. Acreditamos que os médicos que encaminham os casos para serem atendidos pela equipe de psicólogos têm uma percepção da demanda para atendimento psicológico, principalmente frente às doenças em que o componente emocional é relevante, tanto no aparecimento como no enfrentamento, bem como em situações em que sintomas de maior fragilidade psíquica, como a depressão, já se fazem presentes. Também podem pressupor que psicólogos sejam mais bem preparados para manejar sofrimentos e elementos de ordem subjetiva com os quais eles próprios não se sintam capazes de lidar (Catani et al., 2012).No que diz respeito ao motivo da solicitação, alguns autores apontam a presença de sintomas depressivos e ansiosos como os principais fatores de encaminhamento a serviços de Psicologia, muitas vezes associados a uma reação de ajustamento à situação vivida (Carvalho \& Lustosa, 2008; Nakabayashi et al., 2010).

Esses sintomas, em conjunto (ansiedade e depressão), totalizaram $25 \%$ das solicitações de avaliação psicológica no estudo de Osório (2004) e 43\% no estudo de Santos et al. (2011). No estudo de Gazotti e Prebianchi (2014), $33 \%$ dos pacientes tinham depressão e $28,4 \%$, ansiedade. Apesar da alta prevalência desses sintomas como um todo, acreditamos que sua forma e grau de exteriorização são as variáveis diferenciais quanto à tomada de decisão de pedir ou não ajuda a um profissional da Psicologia. Em nossa caracterização, a ansiedade, a depressão ou ambas foram os motivos mais frequentes $(21,7 \%)$ para o encaminhamento, o que condiz também com a queixa do paciente $(26,9 \%)$ e com o diagnóstico do psicólogo (57,1\%). Riolo et al. (2005) apontam que, em geral, a depressão e a ansiedade acometem mais o sexo feminino, o que é o caso de nossa amostra, e que esses tipos de sintomas são mais facilmente encontrados em hospitais gerais.

Em relação ao alto índice geral de solicitações para atendimento de pacientes identificados com humor depressivo, podemos pensar que as respostas do indivíduo à doença e/ou à internação podem acarretar mudanças no seu autoconceito e na sua autoimagem, bem como alterações em seu estilo de vida e na dinâmica dos relacionamentos familiares e sociais. Quando ocorre a permanência prolongada do paciente no hospital, esta pode não apenas ser consequência direta da doença, mas também das relações patológicas estabelecidas no ambiente hospitalar, tornando o tratamento mais difícil (Andreoli \& Mari, 2002; Gazotti \& Prebianchi, 2014).

No que diz respeito às intervenções realizadas, a modalidade de atendimento psicoterápico, em geral individual, aparece na literatura como a mais utilizada, apesar de ser reconhecida a crescente necessidade de criação de novas possibilidades de atuação para a área da saúde. Apesar disso, autores como Osório (2004) salientam a importância dessa prática, que favorece a diminuição da ansiedade e de outras sintomatologias através de informações e modificações de condutas e pensamentos, evidenciando a capacidade e eficácia do profissional psicólogo e de suas técnicas na resolução dos problemas diante dos quais foi solicitado. Entretanto, em nosso estudo, o atendimento psicoterápico embora realizado, na maioria dos casos (56,2\%), como decorrência da realização das avaliações, é sobrepujado por outras duas modalidades de intervenção que aparecem como mais frequentes: a avaliação psicológica $(75,8 \%)$ e o atendimento ao familiar ou cuidador $(77,8 \%)$.

A avaliação psicológica é uma atribuição exclusiva do psicólogo e compete a esse profissional responder pela identificação, descrição e explicação a respeito do funcionamento psicológico humano em qualquer contexto. 
Não há como imaginar o trabalho do psicólogo na saúde sem antes existir uma investigação baseada em referenciais teóricos e metodológicos, que norteie a compreensão de determinado fenômeno psíquico e o planejamento da intervenção. Partimos da importância inicial de uma avaliação cuidadosa do estado emocional, dos mecanismos psíquicos envolvidos, da compreensão a respeito da doença, dos tratamentos realizados, da presença de recursos de enfrentamento diante da situação vivida, do vínculo com a equipe que assiste e da rede de apoio familiar e/ou social. A avaliação viabiliza a formulação de um diagnóstico psicológico, no intuito de compreender dinamicamente o significado da doença na vida do paciente. É importante entender sua relação com a doença, o modo como adoeceu, quão enfermo ele se encontra e como a doença lhe serve. A partir desse entendimento, poderemos construir conclusões lógicas a respeito da terapêutica mais apropriada para o caso.

Entendemos que, no âmbito institucional da saúde, a avaliação psicológica é uma tarefa imprescindível na prática do profissional. É a partir desse instrumental que argumentaremos nosso parecer a respeito de determinado caso, que sustentaremos nossas condutas ou que encontraremos subsídios para amparar nossas decisões e considerações diante da equipe multiprofissional e da instituição, especialmente quando há opiniões divergentes (Lopes \& Amorim, 2006).

Com relação à alta frequência de atendimentos a familiares, levamos em conta que a doença de um membro de uma família é uma situação de crise a ser administrada, que gera sobrecarga e sofrimento emocional, desequilibra o sistema familiar como um todo e desorganiza as relações interpessoais. $\mathrm{O}$ adoecimento de um membro altera $\mathrm{o}$ cotidiano da família, de modo a afetar a dinâmica e o estado emocional dos demais, uma vez que os eventuais problemas financeiros, o medo da morte, o sofrimento do doente e o do próprio familiar se fazem presentes para todos. Estão implícitas mudanças afetivas e instrumentais em tempo curto, contínuas adaptações e mudanças de papéis, que levam a tensões crescentes em quem presta os cuidados com risco de exaustão.
Dessa forma, o familiar deve ser visto como paciente secundário, por estar angustiado e inseguro frente à realidade vivenciada e precisar da oportunidade de expressar seus sentimentos e falar sobre a doença, seus medos e fantasias. O psicólogo tem, assim, uma oportunidade única de evidenciar e mobilizar os recursos de que a família dispõe para a readaptação (Bousso \& Angelo, 2001; Domingues et al., 2013).

Além desses aspectos, em muitas situações, o familiar ou cuidador é considerado um aliado indispensável para a equipe, que, em muitos momentos, vê-se envolta em uma trama familiar que dificulta seu trabalho. Sendo uma fonte constante de informações sobre o paciente, o familiar pode auxiliar o tratamento, dependendo do nível de segurança e confiança que tenha nos profissionais envolvidos. Tranquila e bem informada, a família tende a apoiar o paciente de modo adequado e qualitativo, além de colaborar com a equipe responsável pelo caso (Haberkorn \& Bruscato, 2008). Esse entendimento é o que motivou o atendimento e a orientação ao familiar, que apareceu como a intervenção mais frequente neste estudo, alcançando 77,8\%.

Alguns outros estudos também apontam que a maioria das solicitações $(53,2 \%)$ envolve o atendimento aos familiares e cuidadores devido a questões relacionadas principalmente às dificuldades de adaptação, ao manejo e acomodação aos períodos de crise, à estabilidade ou progressão diante das doenças crônicas e ao impacto do prognóstico reservado e do óbito. As intervenções com familiares são consideradas de extrema importância para os autores, por oferecer um espaço de expressão de medos, fantasias, angústias e trabalhar a percepção da família sobre as habilidades e potencialidades do paciente, a fim de evitar ajustamentos disfuncionais, como superproteção, ou, ainda, que as relações permaneçam enclausuradas na doença, prejudicando a dinâmica e a qualidade das relações (Balieiro \& Cerveny, 2004; Ribeiro et al., 2008). Desse modo, pode-se pensar que, no cenário estudado, a intervenção psicológica seria um recurso fundamental para favorecer o período de tratamento do paciente, a relação entre a equipe de saúde, o paciente e seus familiares e entre os próprios profissionais da equipe de saúde.

\section{CONCLUSÃO}

Foi caracterizado o atendimento psicológico em um complexo prestador de serviços de saúde ao SUS. O perfil sociodemográfico dos pacientes atendidos revelou maior porcentagem de indivíduos do sexo feminino e a predominância de quadros de ansiedade e depressão. As intervenções mais utilizadas foram a avaliação psicológica $\mathrm{e} o$ atendimento do familiar/cuidador.

Além de caracterizar a população atendida, os resultados apontaram que, nas unidades de saúde onde ocorreu o estudo, a inserção da Psicologia nas equipes profissionais é um fato reconhecido. O estudo também detalhou um serviço que está em constante desenvolvimento e que cresce consideravelmente na oferta de atendimento psicológico para comunidades usuárias do SUS. Além disso, constatouse um molde de trabalho para os profissionais da Psicologia nos âmbitos da saúde.

Estudos rotineiros de caracterização da assistência são essenciais para o monitoramento do desempenho dos serviços, pois permitem verificar se as ações ou estratégias utilizadas estão alcançando os objetivos estipulados ou se é 
necessário seu aprimoramento para desenvolver uma atuação mais criativa e mais próxima das demandas das unidades de saúde. O conhecimento produzido por esses estudos pode melhorar a organização da assistência psicológica, estabelecendo a atenção voltada aos atributos dos indivíduos, além de permitir a identificação de grupos populacionais mais vulneráveis, promover novos questionamentos e impor alterações, tanto na atuação quanto na formação dos psicólogos. Tal conhecimento pode melhorar a qualidade da assistência ao paciente através da integração de práticas e cuidados, possibilitando o entendimento amplo da dinâmica da situação, reaproximando o psíquico do somático e contribuindo para o processo de humanização do ambiente e das relações na área da saúde. Num meio tão potencialmente adverso como o campo da saúde, pode-se constituir uma das formas de firmar a presença e a necessidade do psicólogo como membro efetivo de equipes de saúde, além de priorizar o processo de atenção integral e servir de fonte de informação à tomada de decisões sobre o usuário do sistema de saúde (Turra et al., 2012). Além da melhoria da assistência, a caracterização do atendimento também guarda uma relação direta com o encorajamento de pesquisas, destacando a relevância da geração de conhecimentos, métodos e tecnologia que deem suporte ao desenvolvimento ou melhoria das políticas de saúde. Destaca-se também a importância da coleta e sistematização dos dados colhidos para que o trabalho da equipe de saúde possa realmente oferecer trocas e benefícios para os interessados.
No entanto, o formulário utilizado, dentre outras falhas, não contemplou a percepção de satisfação do usuário, o que impossibilitou a obtenção da informação direta sobre os resultados do atendimento psicológico realizado, o que revela as limitações ainda envolvidas nesse Serviço e prejudica a verificação da efetividade das atuações e repercussões dos serviços prestados e o aperfeiçoamento das intervenções realizadas. De qualquer forma, conforme preconizado por Gazotti e Prebianchi (2014), o estudo realizado serviu para uma revisão de práticas e conhecimentos que só pode ser concretizada a partir de uma postura de constante investigação e uma melhor instrumentalização técnica fundamentada em desenvolvimentos teóricos consistentes para subsidiar as ações que ampliam os limites tradicionais, possibilitando a utilização de recursos que se coadunam com as novas exigências desse alargamento de fronteiras profissionais dos psicólogos.

Levando em conta a importância do atendimento psicológico como atividade interdisciplinar que assegura a humanização do atendimento ao paciente, estudos continuados são necessários sobre o tema. Estudos com esse enfoque podem servir tanto para aumentar a visibilidade dessa atividade profissional, contribuindo assim para a consolidação de sua prática, como para produzir conhecimentos que possam ser utilizados pelos profissionais $\mathrm{e}$, por conseguinte, oferecidos à população que usufrui dos serviços de Psicologia (Gazotti \& Prebianchi, 2014).

\section{REFERÊNCIAS}

Amaral, V. A., Uwagoya, K. S. H., Pinto, L. A., Kioroglo, P. S., Pozzani, A. F., Carvalho, D. C., Sasso, L. G. C., Silva, L. M. S., Bonsaver, M., \& Rosa, N. B. M. (2012). Atendimento psicológico em enfermarias. In W. L. Bruscato(Org.), $A$ Psicologia na saúde da atenção primária à alta complexidade: O modelo de atuação da Santa Casa de São Paulo (pp. 135148). Casa do Psicólogo.

Andreoli, P. B. A., \& Mari, J. J. (2002). Assessment of a consultation liaison psychiatry and psychology health care program. Revista de Saúde Pública, 36(2), 222-229. https://doi.org/10.1590/ S0034-89102002000200015

Balieiro, C. R. B., \& Cerveny, C. M. O. (2004). Família e doença. In C. M. O. Cerveny. (Org.), Família e comunicação, divórcio, mudança, resiliência, deficiência, lei, bioética, doença, religião e drogadicção (pp. 147-161). Casa do Psicólogo.

Baremblitt, G. F. (2002). Compêndio de análise institucional e outras correntes: Teoria e prática. Instituto Felix Guattari.

Borim, F. S., Barros, M. B., \& Botega, N. J. (2013). Common mental disorders among elderly individuals: A populationbased study in Campinas, São Paulo State, Brazil. Cadernos de Saúde Coletiva, 29(7), 1415-1426. https://doi.org/10.1590/ S0102-311X2013000700015

Botega, N. J. (Org.). (2012). Prática psiquiátrica no hospital geral: Interconsulta e emergência. Artmed.

Botega, N. J., \& Smaira, S. I. (2012). Morbidade psiquiátrica no hospital geral. In N. J. Botega (Org.), Prática psiquiátrica no hospital geral: Interconsulta e emergência (pp. 31-42). Artmed.

Bousso, R. S., \& Angelo, M. (2001). Buscando preservar a integridade da unidade familiar: A família vivendo a experiência de ter um filho na UTI. Revista da Escola de Enfermagem da USP, 35(2), 172-179. https://doi.org/10.1590/ S0080-62342001000200012

Braga, A. P. S. M., Boldrini, C. R., \& Machado, T. P. F. (2012). Neuropsicologia nas doenças neurológicas. In W. L. Bruscato (Org.), A Psicologia na saúde da atenção primária à alta complexidade: O modelo de atuação da Santa Casa de São Paulo (pp. 219-230). Casa do Psicólogo.

Brasil. (1990). Lei $n^{\circ} 8.080$ de 19 de setembro de 1990. Senado.

Bruscato, W. L. (2006). A Psicologia no hospital da misericórdia: Um modelo de atuação. In W. L. Bruscato, C. Benedetti, \& S. R. A. Lopes (Orgs), A prática da Psicologia Hospitalar na Santa Casa de São Paulo: Novas páginas em uma antiga história (pp. 17-31). Casa do Psicólogo.

Bruscato, W. L. (2012). 20 anos de Psicologia na Misericórdia: nossa missão, nossa visão, nossos valores. In W. L. Bruscato et al. (Orgs.) A Psicologia na saúde da atenção primária à alta complexidade: o modelo de atuação da Santa Casa de São Paulo (pp. 15-43). Casa do Psicólogo.

Carvalho, M. R., \& Lustosa, M A. (2008). Interconsulta psicológica. Revista da Sociedade Brasileira de Psicologia Hospitalar, 11(1),31-47.http://pepsic.bvsalud.org/scielo.php?script=sci_ar ttext\&pid=S1516-08582008000100004

Castro, E. K., \& Bornholdt, E. (2004). Psicologia da Saúde x Psicologia Hospitalar: Definições e possibilidades de inserção profissional. Psicologia Ciência e Profissão, 24(3), 48-57. https://doi.org/10.1590/S1414-98932004000300007

Catani, J., Juhas, T. R., Santos, N. O., Moretto, M. L. T., \& Lucia, M. C. S. (2012). Motivos de encaminhamento à psicologia 
e a escuta da demanda médica no serviço de imunologia e alergia. Psicologia Hospitalar, 10(2), 33-50. http://pepsic. bvsalud.org/scielo.php?script $=$ sci_arttext\&pid $=\mathrm{S} 1677$ $74092012000200004 \& \operatorname{lng}=\mathrm{pt \& nrm}=\mathrm{iso} \& \operatorname{lng}=\mathrm{pt}$

Chiavone, P. A., \& Sens, Y. A. S. (2003). Evaluation of Apache II System among intensive care patients at a teaching hospital. São Paulo Medical Journal, 121(2), 53-57. https://doi. org/10.1590/S1516-31802003000200004

Domingues, G. R., Alves, K. O., Carmo, P. H. S., Galvão, S. S., Teixeira S. S., \& Baldoino, E. F. (2013). A atuação do psicólogo no tratamento de pacientes terminais e seus familiares. Psicologia Hospitalar, 11(1), 2-24. http:// pepsic.bvsalud.org/scielo.php?script $=$ sci_arttext\&pid $=\mathrm{S} 1677-74092013000100002$

Fortes, S. L. (2012). O paciente com dor. In N. J. Botega (Org.), Prática psiquiátrica no hospital geral: Interconsulta e emergência (pp. 339-351). Artmed.

Garcia Jr, C., \& Zimmerman, P. R. (2012). Falência e transplante de órgãos. In N. J. Botega (Org.), Prática psiquiátrica no hospital geral: Interconsulta e emergência (pp. 298-313). Artmed.

Gazotti, T.C., \& Prebianchi, H. B. (2014). Caracterização da interconsulta psicológica em um hospital geral. Psicologia Teoria e Prática 16(1), 18-30. http://pepsic.bvsalud.org/scielo. php?script $=$ sci_arttext\&pid=S1516-36872014000100002

Haberkorn, A. Atuação psicológica na UTI. (2006). In W. L. Bruscato (Org.), A prática da Psicologia Hospitalar na Santa Casa de São Paulo: Novas páginas em uma antiga história (pp. 99-107). Casa do Psicólogo.

Haberkorn, A., \& Bruscato, W. L. (2008). Qualidade de vida e internação em UTI. In E. Knobel, P. B. A. Andreoli, \& M. R. Erlichman (Orgs.), Psicologia e humanização: Assistência aos pacientes graves (pp. 125-132). Atheneu.

IBGE. (2015). Projeção da população brasileira. http://www. ibge.gov.br/

Kitayama, M. M. G., \& Bruscato, W. L. (2008). Abordagem psicológica da dor do paciente grave. In E. Knobel, P. B. A. Andreoli, \& M. R. Erlichman (Orgs.), Psicologia e humanização: Assistência aos pacientes graves (pp. 133-147). Atheneu.

Lopes, S. R. A., \& Amorim, S. F. (2006). Avaliação psicológica no Hospital Geral. In W. L. Bruscato (Org.), A prática da Psicologia Hospitalar na Santa Casa de São Paulo: Novas páginas em uma antiga história (pp. 53-68). Casa do Psicólogo.

Lucchese, R., Sousa, K., Bonfin, S. P., Vera, I., \& Santana, F. R. (2014). Prevalência de transtorno mental comum na atenção primária. Acta Paulista de Enfermagem, 27(3), 200-207. https://doi.org/10.1590/1982-0194201400035

Machado, N. P., \& Nogueira, L. T. (2008). Avaliação da satisfação dos usuários de serviços de Fisioterapia. Revista Brasileira de Fisioterapia, 12(5), 401-408. https://doi.org/10.1590/S141335552008000500010

Matarazzo, J. D. (1982). Behavior health's challenge to academic, scientific and professional psychology. American Psychologist, 37, 1-14. https://doi.org/10.1037//0003-066x.37.1.1

Mendoza-Sassi, R., Béria, J. U., \& Barros, A. J. D. (2003). Outpatient health service utilization and associated factors: a population-based study. Cadernos de Saúde Pública, 37(3), 372-378. https://doi.org/10.1590/S0034-89102003000300017

Michelone, A. P. C., \& Santos, V. L. C. G. (2004). Qualidade de vida de adultos com câncer colorretal com e sem ostomia. Revista Latino-Americana de Enfermagem, 12(6), 875-883. https:// doi.org/10.1590/S0104-11692004000600005

Moreira, J. K., Bandeira, M., Cardoso, C. S., \& Scalon, J. D. (2011). Prevalence of common mental disorders in the population attended by the Family Health Program. Jornal Brasileiro de
Psiquiatria, 60(3), 221-226. https://doi.org/10.1590/S004720852011000300012

Nakabayashi, T. I. K., Guerra, K. A., Souza, R. M., Loureiro, S. R., Contel, J. O. B., Cabrera, C. C., Hallak, J. E. C., Osório, F. L., Leal, C. G., Rufino, A. C. T. B. F., \& Crippa, J. A. S. (2010). Comparação entre solicitações psiquiátricas de dois hospitais gerais universitários brasileiros: uso do protocolo de registro de interconsulta em saúde mental. Cadernos de Saúde Pública, 26(6), 1246-1260. https://doi.org/10.1590/ S0102-311X2010000600017

Oliveira, E. R. A., Fiorin, B. H., Lopes, I. J., Gomes, M. J., Coelho, S. O., \& Morra, J. S. (2011). Interdisciplinaridade, trabalho em equipe e multiprofissionalismo: Concepções dos acadêmicos de enfermagem. Revista Brasileira de Pesquisa em Saúde, 13(4), 28-34. https://doi.org/10.21722/rbps.v0i0.2996

Osório, F. L. (2004). O psicólogo interconsultor na Enfermaria de Pneumologia de um hospital escola: Caracterização das solicitações de atendimento psicológico. Medicina, 37, 76-83. https://doi.org/10.11606/issn.2176-7262.v37i1/2p76-83

Ribeiro, C. A., Almeida, F. A., \& Borba, R. I. (2008). A criança e o brinquedo no hospital. In F. A. Almeida \& A. L. Sabatés. (Orgs.), Enfermagem pediátrica: A criança, o adolescente e sua familia no hospital (pp. 65-77). Manole.

Ribeiro, M. C. S. A., Barata, R. B., Almeida, M. F., \& Silva, Z. P. (2006). Perfil sociodemográfico e padrão de utilização de serviços de saúde para usuários e não usuários do SUS - PNAD 2003. Ciência e Saúde Coletiva, 11(4), 1011-1022. https://doi. org/10.1590/S1413-81232011001000016

Riolo, S. A., Nguyen, T. A., Greden, J. F., \& King, C. A. (2005). Prevalence of depression by race/ethnicity: Findings from the National Health and Nutrition Examination Survey III. American Journal of Public Health, 95(6), 998-1000. https:// doi.org/10.2105/AJPH.2004.047225

RIPSA. (2015). Indicadores e Dados Básicos para a Saúde - IDB 2008. http://tabnet.datasus.gov.br/

Ruiz, G. (2015). Quem usa o Sistema Único de Saúde? Portal DSS Brasil, 2012. http://dssbr.org/site/?p=9534\&preview=true

Santos, N. C. A., Slonczewski, T., Prebianchi, H. B., Oliveira, A. G., \& Cardoso, C. S. (2011). Interconsulta psicológica: Demanda e assistência em hospital geral. Psicologia em Estudo, 16(2), 325-334. https://doi.org/10.1590/S1413-73722011000200016

Silva, Z. P., Ribeiro, M. C. S. A., Barata, R. B., \& Almeida, M. F. (2011). Perfil sociodemográfico e padrão de utilização dos serviços de saúde do Sistema Único de Saúde (SUS), 20032008. Ciência e Saúde Coletiva, 16(9), 3807-3816. https://doi. org/10.1590/S1413-81232011001000016

Sistema de Informação Santa Casa (SISC). (2015). Dados estatísticos. http://intranet/sisc/acesso/cadpgv

Travassos, C., \& Martins, M. (2004). Uma revisão sobre os conceitos de acesso e utilização de serviços de saúde. Cadernos de Saúde Pública, 20(Supl. 2), S190-S198. https://doi.org/10.1590/ S0102-311X2004000800014

Traverso-Yépez, M. (2001). A interface Psicologia Social e Saúde: Perspectivas e desafios. Psicologia em Estudo, 6(2), 49-56. https://doi.org/10.1590/S1413-73722001000200007

Turra, V., Almeida, F. F., Doca, F. N. P., \& Costa Junior, A. L. (2012). Protocolo de atendimento psicológico em saúde orientado para o problema. PSICO, 43(4), 500-509. http://revistaseletronicas. pucrs.br/ojs/index.php/revistapsico/article/view/10625

Vanni, M. G., \& Maggi, A. (2005). O que demanda à Psicologia na Rede Pública de Saúde em Caxias do Sul? PSICO, 36(3), 299-309. http://revistaseletronicas.pucrs.br/ojs/index.php/ revistapsico/article/viewFile/1401/1101

Zimmerman, P. R., \& Bertuol, C. S. (2012). O Paciente na UTI. In N. J. Botega (Org.), Prática Psiquiátrica no Hospital Geral: Interconsulta e Emergência (pp. 331-338). Artmed. 